\title{
Estudio descriptivo sobre las diferencias de género en el estrés académico derivado del contexto COVID-19 en población universitaria española Descriptive study on gender differences in academic stress derived from the COVID-19 context in a Spanish university population \\ *Adrià Marco-Ahulló, **Israel Villarrasa-Sapiña, ***Gonzalo Monfort-Torres *Universidad Católica de Valencia (España) **Universidad de Valencia (España). ***Florida Universitaria (España)
}

Resumen. En la actualidad, la crisis sanitaria derivada de la aparición del COVID-19 ha repercutido de forma significativa en las vidas de la población, la cual ha experimentado muchos cambios en un espacio muy corto de tiempo. Los estudiantes universitarios son una de las poblaciones que más se han tenido que adaptar a este contexto pandémico, lo cual puede haber generado nuevas situaciones de estrés, afectando a su salud mental. El objetivo principal de este estudio es analizar los niveles de estrés académico de una muestra de estudiantes universitarios, así como identificar los principales estresores, sintomatología asociada al estrés y las estrategias de afrontamiento adoptadas por cada género. La muestra estuvo compuesta por 351 estudiantes universitarios (211 mujeres) a los que se les facilitó el Inventario Sistémico Cognoscitivista (SISCO) para el estudio del Estrés Académico adaptado al contexto de la crisis por COVID-19. Los resultados hallados mostraron diferencias significativas entre los niveles de estrés y variables relacionadas con este entre hombres y mujeres, siendo las estudiantes las que mostraron mayores niveles de estrés $(\mathrm{t}(260.077)=-4.801, \mathrm{p} £ 0.01, \mathrm{r}=0.29)$. Además, también se hallaron diferencias en la elección de las estrategias de afrontamiento utilizadas por hombres y mujeres. En conclusión, los resultados del presente trabajo mostraron diferencias estadísticamente significativas entre los niveles de estrés de los estudiantes universitarios dependiendo de su género.

Palabras clave: COVID-19; estrés; universidad; pandemia.

\begin{abstract}
Currently, the health crisis resulting from the appearance of COVID-19 has had a significant impact on the lives of the population, which has undergone many changes in a very short space of time. University students are one of the populations that have had to adapt the most to this pandemic context, which may have generated new stressful situations, affecting their mental health. Therefore, the main objective of this study was to analyse the levels of academic stress in this population, as well as to identify the main stressors, symptomatology associated with stress and the coping strategies adopted by each gender. The total study sample consisted of 351 university students (211 women) who were provided with the Systemic Cognitive Inventory for the Study of Academic Stress (SISCO) adapted to the context of the crisis by COVID-19. The results showed significant differences in stress levels and stress-related variables between men and women, with female students showing higher levels of stress $(\mathrm{t}(260.077)=-4.801, \mathrm{p} £ 0.01, \mathrm{r}=0.29)$. In addition, differences were also found between in the election of the coping strategies used by men and women. In conclusion, the results of the present study showed statistically significant differences between the stress levels of university students depending on their gender. Furthermore, the main stressors, stress-associated symptomatology and coping strategies adopted by each gender were identified.
\end{abstract}

Keywords: COVID-19; stress; university; pandemic.

\section{Introducción}

La aparición del COVID-19 ha supuesto un cambio en las rutinas diarias de la mayor parte de la población mundial. Durante los últimos tiempos se han aplicado diversas restricciones con el fin de contener la propagación del virus, afectando a diferentes dimensiones del comportamiento humano.

La investigación acerca del impacto causado por la

Fecha recepción: 26-04-21. Fecha de aceptación: 09-09-21

Israel Villarrasa-Sapiña

israel.villarrasa@unir.net crisis provocada por la COVID-19 está teniendo una rápida expansión, debido al interés por comprender cómo afectan a las personas las novedosas situaciones que se han sucedido. Es por ello que, los efectos de los cambios propiciados por la pandemia (familiares enfermos, enfermedad propia, confinamientos en el hogar, cierres, restricciones horarias y de movilidad, regulaciones en el ámbito laboral y educativo), están siendo un tema candente de estudio (Cullen, Gulati, y Kelly 2020; Islam et al. 2020; Pfefferbaum y North 2020; Vigo et al. 2020; Vindegaard y Benros 2020).

Aunque la pandemia ha tenido un gran impacto a nivel mundial, cada uno de los países ha adoptado dife- 
rentes políticas para hacer frente a esta crisis sanitaria. Por tanto, también son diferentes las realidades vividas, así como los posibles efectos derivados de esta situación. En el caso del territorio español, en un primer momento se impuso el confinamiento el 15 de marzo del 2020, finalizando el 21 de junio de 2020 coincidiendo con el fin del estado de alarma. A nivel educativo, durante este periodo de tiempo, las instituciones educativas (escuelas, institutos y universidades) se mantuvieron cerradas. De esta manera, viéndose imposibilitada la presencialidad, la educación tuvo que sostenerse en las nuevas tecnologías para poderse llevar a cabo, dando paso a nuevos casos de desigualdad educativa por esta razón (Diez Gutierrez y Gajardo Espinoza 2020; Jiménez Hernández, Cáceres-Muñoz, y Martín-Sánchez 2021). Con la llegada del nuevo curso académico 2020 / 21 , se reabrieron de nuevo los centros educativos con nuevos protocolos para evitar la transmisión del COVID-19, estableciéndose en algunos casos la semipresencialidad.

Impartir docencia durante este contexto pandémico está resultando un reto tanto para profesores como para alumnos, los cuáles han experimentado cambios bruscos en su educación en un corto periodo de tiempo. Es presumible que dichas variaciones hayan contribuido a la aparición de estrés académico, el cual se ha definido como una reacción de activación fisiológica, emocional, cognitiva y conductual ante estímulos y eventos relacionados con el ámbito académico (García y Zea 2011).

Por ello, una de las poblaciones en las que la literatura ha puesto el foco de atención es la formada por los estudiantes (Benham 2020; Bono, Reil, y Hescox 2020; Husky, Kovess-Masfety, y Swendsen 2020; Moawad 2020). Los principales cambios asociados a la pandemia que ha tenido que asumir esta población han sido el confinamiento en el hogar, y derivado de esta medida, cambios en la metodología de enseñanza de manera generalizada (Adnan y Anwar 2020; Agarwal y Kaushik 2020; Bao 2020). Este cambio en las costumbres y hábitos ha llevado consigo una serie de desórdenes a nivel de salud mental, tales como incrementos referenciados en los niveles de estrés, ansiedad y, en algunos casos, pensamientos suicidas (Son et al. 2020; Wang et al. 2020). Dichas afecciones se activaron durante el periodo del confinamiento, aunque muchas han perdurado más allá del mismo, necesitando de ayuda profesional (Rogowska, Kuœnierz, y Bokszczanin 2020).

La actual evidencia científica presenta diversos posicionamientos en la definición de los niveles de estrés en función del género y su influencia en el rendimiento académico. Brougham et al. (2009) observaron resultados que mostraron que las mujeres universitarias padecían un mayor nivel general de estrés y un mayor uso de estrategias de afrontamiento centradas en la emoción que los hombres universitarios. Por su parte, Backoviæ et al. (2012) hallaron que las estudiantes evaluaron su estado de salud física y su nivel de estrés general como peores en comparación con los hombres. Cabanach et al. (2013) alcanzó resultados en la línea de los de Brougham et al. (2009), poniendo de manifiesto que los hombres recurren en mayor medida a las estrategias de reevaluación positiva y planificación como medidas de afrontamiento de situaciones académicas problemáticas; mientras que las mujeres optan fundamentalmente por la búsqueda de apoyo. No obstante, aunque al parecer las mujeres tengan un nivel de estrés académico, en general, más elevado que el de los hombres, hay estudios que indican que presentan un manejo más eficiente del tiempo, priorizan metas, planean y se organizan mejor (Misra, Crist, y Burant 2003), y su mayor percepción de estrés no se acompaña con un descenso de su rendimiento académico (Pomerantz, Altermatt, y Saxon 2002).

Después de todo lo expuesto anteriormente, y observando los cambios forzosos que se han producido a nivel educativo para poder continuar con la docencia en periodo pandémico, la comunidad científica debe seguir recabando información del efecto de los confinamientos o los cambios de hábitos en la rutina académica puedan tener sobre la salud de la población estudiantil. Además, se debe tener en especial consideración la variable género, ya que es uno de los factores que según la literatura influyen en la percepción del estrés y en las estrategias de afrontamiento del estrés académico (Eckles 1987; Matud 2004; Miller y Kirsch 1987).

Es por ello por lo que, el objetivo principal del presente trabajo fue describir el grado autoreportado de estrés académico, así como los principales estresores (ES), sintomatología asociada al estrés (SI) y estrategias de afrontamiento (EA) adoptadas por la población de estudiantes universitarios, en función del género, durante el periodo de influencia de las medidas derivadas del COVID-19.

\section{Material y métodos}

\section{Diseño del estudio y participantes}

Un estudio transversal con diseño descriptivo comparativo fue llevado a cabo para detectar el estrés académico generado por la pandemia del COVID-19 en- 
tre los estudiantes universitarios de España. Para ello, se realizó un muestreo no probabilístico por cuotas, puesto que el cuestionario fue distribuido en formato electrónico por cinco universidades españolas que facilitaron su difusión. Dicho muestreo se aplicó atendiendo a los criterios exclusión. Es decir, si el alumnado universitario no atendía a ningún factor exclusivo (los cuales se indican a continuación), podía participar en el estudio. Por último, cabe destacar que la participación en el estudio era totalmente voluntaria.

La muestra total del estudio estuvo compuesta por 351 alumnos universitarios. Los participantes fueron reclutados de diferentes universidades españolas y los criterios de exclusión fueron: i) no estar matriculado en un grado o máster universitario en España, ii) tener menos de 18 años, y iii) padecer alguna enfermedad o patología cognitiva que no permita entender adecuadamente el cuestionario.

\section{Procedimiento}

El cuestionario utilizado para llevar a cabo el estudio fue el Inventario Sistémico Cognoscitivista (SISCO) para el estudio del Estrés Académico adaptado al contexto de la crisis por COVID-19 (Alania-Contreras et al. 2020). Este es un cuestionario adaptado del Inventario SISCO - SV, de Barraza-Macías (2007, 2018), al que se le han añadido 2 ítems en la tercera dimensión. La validez de dicho cuestionario se representa con los coeficientes de concordancia V de Aiken mayor de 0.75, de correlación de $r$ de Pearson corregida mayor de 0.2 y de consistencia interna alfa de Cronbach superior a 0.88. Dicho cuestionario fue pasado al finalizar el primer cuatrimestre del curso académico 2020/21, para conocer el estrés generado tras unos meses de convivir académicamente con la situación generada por el COVID19.

El cuestionario constaba de 49 preguntas. La primera era una pregunta independiente y discriminatoria, donde los participantes solo podían elegir una respuesta, «sí» o «no». Es decir, a los participantes se les preguntaba si durante el transcurso del cuatrimestre habían sufrido momentos de preocupación o nerviosismo (i.e., estrés) a causa del Covid-19 y, si contestaban «sí», continuaban respondiendo el resto del cuestionario. En cambio, si respondían «no», finalizaban el cuestionario.

La segunda pregunta también era independiente y servía para conocer el nivel de nerviosismo y preocupación que percibían los participantes, siendo 1 el nivel mínimo de estrés y 5 el máximo. El nivel de estrés se diferencia entre leve (de 0 a 48\%, es decir, de 0 a 2.38) medio (de 49\% a 60\%, es decir, de 2.39 a 3.0) y severo (de 61\% a 100\%, es decir, de 3.1 a 5). Aunque, el autor menciona que esto puede variar dependiendo de la población y que, si los investigadores lo prefieren, estos baremos pueden cambiar a leve (de 0 a 33\%), medio (de 34\% a 66\%) y severo (de 67\% a 100\%) (BarrazaMacías 2018).

Por último, las 47 preguntas restantes del cuestionario estaban divididas en 3 dimensiones: i) estresores (ES), ii) síntomas (SI) y iii) estrategias de afrontamiento (EA). Las dos primeras estaban formadas por 15 preguntas cada una y la tercera con 17. Todas estas preguntas estaban compuestas por seis opciones de respuesta en una escala de tipo Likert, desde 0 a 5 , donde 0 es el valor más bajo y 5 el más alto (i.e., 0 nunca, 1 casi nunca, 2 raras veces, 3 algunas veces, 4 casi siempre y 5 siempre). La dimensión SI, a su vez, estaba dividida en 3 subdimensiones: reacciones físicas (preguntas 1 a 6), reacciones psicológicas (preguntas 7 a 11) y reacciones comportamentales (preguntas de 12 a 15).

\section{Análisis estadístico}

El análisis estadístico se realizó mediante el programa SPSS 24 (IBM, Armonk, USA). En primer lugar, tras recopilar todos los datos de los cuestionarios, se obtuvieron los datos descriptivos y las frecuencias de cada variable. Además, puesto que el número de participantes era elevado, se asumió la distribución normal de la muestra.

En segundo lugar, se atendió a la pregunta 1 para conocer en número de participantes que sufrieron o no estrés durante el primer cuatrimestre. Aquellos que no tenían estrés fueron excluidos de los siguientes pasos del análisis.

Los participantes se dividieron por géneros (i.e., mujeres y hombres) y, en aquellos que respondieron «sí» en la pregunta 1, se calcularon los estadísticos de centralidad de cada una de las dimensiones del cuestionario (ie., ES, SI y EA), así como de las subdimensiones de la dimensión SI (i.e., reacciones físicas, psicológicas y comportamentales). Por último, se aplicó una prueba t de Student para comparar los resultados del cuestionario, cogiendo el factor de género como variable independiente. El nivel de significación se fijó en $\mathrm{p}<0.05$ para todos los análisis.

Cabe destacar que, en esta muestra, no hubo necesidad de imputar o substituir los datos perdidos ya que en ningún caso sobrepasó el 5\% (Littlewood, 2014). El ítem con mayor cantidad de datos perdidos fue la pregunta $S I$ 5 con un $3.1 \%$. 


\section{Resultados}

Del total de 351 participantes, 240 tenían entre 18 y 24 años (media $=20.67$ y desviación estándar $=0.50)$, ambos inclusive, y 111 indicaron tener 25 años o más. Estas cantidades se dividieron entre sexos, siendo 104 hombres entre 18 y 24 años (media $=20.83$ y desviación estándar $=1.81$ ) y 36 mayores de 25 años y, 136 mujeres entre 18 y 24 años (media $=20.53$ y desviación estándar $=1.69$ ) y 75 mayores de 25 años.

Mediante la pregunta 1 del cuestionario, se encontró que 29 participantes señalaron que no habían sufrido estrés durante el primer cuatrimestre, por lo que fueron excluidos de los siguientes análisis. Las frecuencias y porcentajes de estos grupos se pueden observar en la Tabla 1.

\begin{tabular}{lccc} 
Tabla 1 \\
Frecuencias y porcentajes de la pregunta 1 \\
\hline \multicolumn{1}{c}{ Sexo } & Estrés "si" & & \\
\hline \multirow{2}{*}{ Hombre } & 124 & Estrés "no" & Total \\
& $(88.6 \%)$ & 16 & 140 \\
Mujer & 198 & $(11.4 \%)$ & \\
& $(93.8 \%)$ & 13 & 211 \\
Total & 322 & $(6.2 \%)$ & \\
& $(91.7 \%)$ & 29 & 351 \\
\hline
\end{tabular}

Los resultados descriptivos obtenidos del resto del cuestionario de aquellos participantes que indicaron que sí tuvieron estrés durante el primer cuatrimestre se muestra en la Tabla 2, donde se puede observar la media y el error estándar de ambos sexos en cada uno de los ítems del cuestionario. Para facilitar la interpreta-

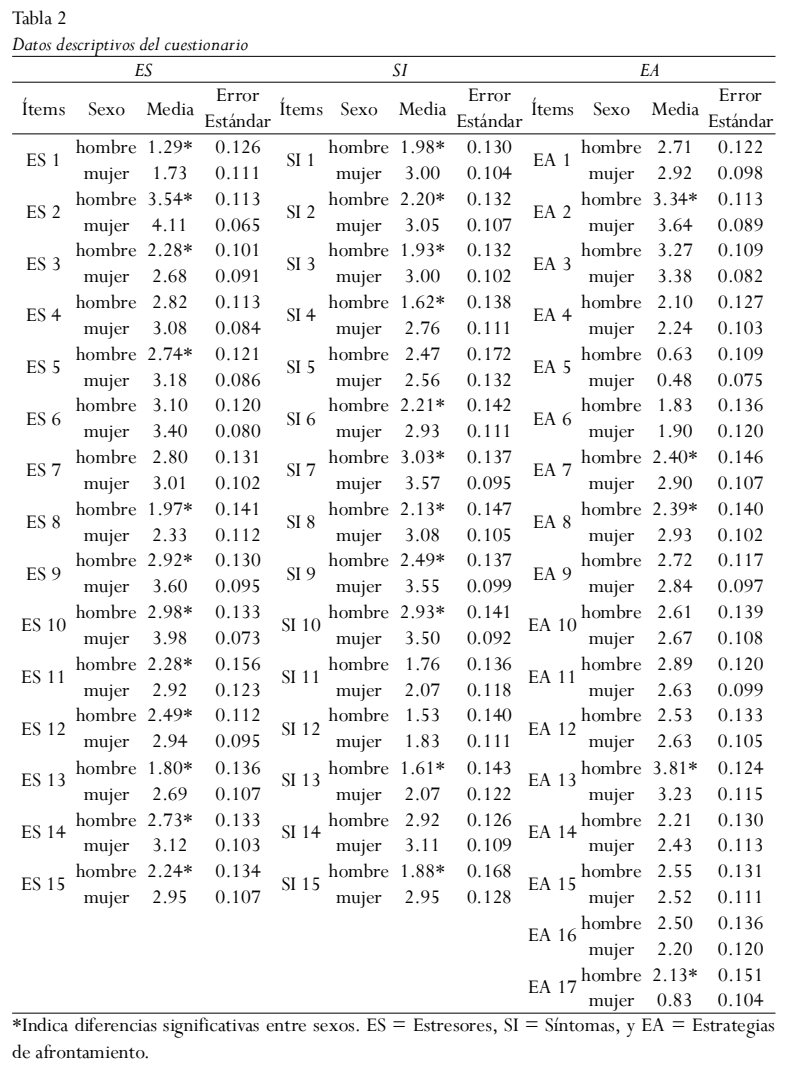

ción de las tablas 2 y 4, las preguntas se han codificado en ítems, como en el artículo de validación (AlaniaContreras et al., 2020), indicando la dimensión de pertenencia (i.e., ES, SI o EA) y el número de pregunta, por orden de aparición en el mismo cuestionario.

Mediante estos datos descriptivos de las preguntas, se analizaron las dimensiones. Los resultados de este estudio mostraron que existe una diferencia significativa entre géneros en el nivel de estrés ( $t(260.077)=$ 4.801, $\mathrm{p} £ 0.01, \mathrm{r}=0.29)$ y en las dimensiones ES $(\mathrm{t}(228.64)=-5.374, \mathrm{p} £ 0.01, \mathrm{r}=0.33)$ y SI $(\mathrm{t}(238.417)=-$ 5.989, $\mathrm{p} £ 0.01, \mathrm{r}=0.36)$, así como en las tres subdimensiones de SI: reacciones físicas $(\mathrm{t}(257.667)=$ 7.024, $\mathrm{p} £ 0.01, \mathrm{r}=0.40)$, psicológicas $(\mathrm{t}(234.678)=$ 5.109, $\mathrm{p} £ 0.01, \mathrm{r}=0.32)$ y comportamentales $(\mathrm{t}(234.678)=-3.553, \mathrm{p} £ 0.01, \mathrm{r}=0.22)$. En cambio, no se mostraron diferencias en la dimensión EA. Los datos descriptivos de este análisis se pueden comprobar en la tabla 3 .

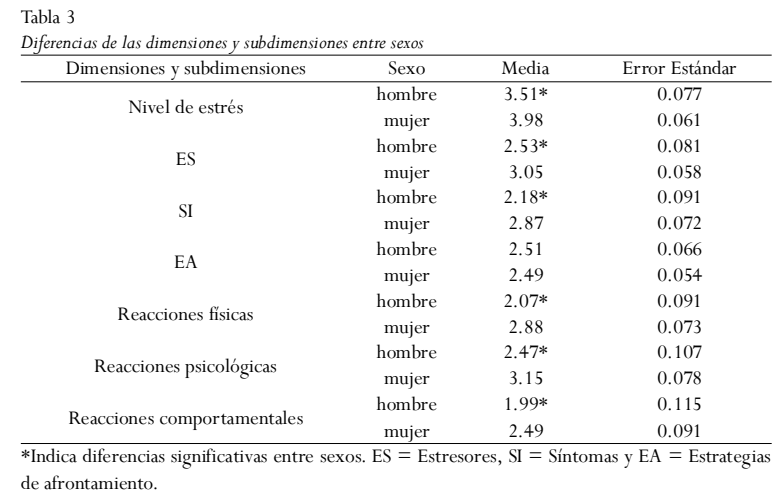

Más concretamente, también se encontraron diferencias significativas entre los diferentes ítems del cuestionario, como se puede observar en la Tabla 4.

Tabla 4

Resultados $t$ de Student en cada item entre sexos

\begin{tabular}{|c|c|c|c|c|c|c|c|c|c|c|c|}
\hline \multicolumn{4}{|c|}{$E S$} & \multicolumn{4}{|c|}{$S I$} & \multicolumn{4}{|c|}{$E A$} \\
\hline Items & $\mathrm{t}$ & $\mathrm{gl}$ & $p$ & Ítems & $\mathrm{t}$ & $\mathrm{gl}$ & $p$ & Items & $\mathrm{t}$ & $\mathrm{gl}$ & $p$ \\
\hline ES 1 & -2.638 & 281.283 & .009 & SI 1 & -6.100 & 257.691 & .000 & EA 1 & -1.350 & 258.460 & .178 \\
\hline ES 2 & -4.351 & 202.743 & .000 & SI 2 & -5.012 & 260.669 & .000 & EA 2 & -2.111 & 256.644 & .036 \\
\hline ES 3 & -2.942 & 286.309 & .004 & SI 3 & -6.429 & 250.376 & .000 & EA 3 & -0.812 & 245.642 & .418 \\
\hline ES 4 & -1.819 & 246.976 & .070 & SI 4 & -6.432 & 259.415 & .000 & EA 4 & -0.839 & 262.031 & .402 \\
\hline ES 5 & -2.973 & 241.473 & .003 & SI 5 & -0.428 & 250.023 & .669 & EA 5 & 1.132 & 229.705 & .259 \\
\hline ES 6 & -2.063 & 224.913 & . 040 & SI 6 & -3.994 & 252.810 & .000 & EA 6 & -0.340 & 75.641 & .734 \\
\hline ES 7 & -1.278 & 258.601 & 202 & SI 7 & -3.230 & 228.491 & .001 & EA 7 & -2.741 & 44 & .007 \\
\hline ES 8 & -2.002 & 263.362 & .046 & SI 8 & -5.230 & 235.520 & .000 & EA 8 & -3.152 & 240.243 & .002 \\
\hline ES 9 & -4.198 & 246.911 & .000 & SI 9 & -6.252 & 239.205 & .000 & EA 9 & -0.781 & 266.042 & .435 \\
\hline ES 10 & -6.618 & 197.273 & .000 & SI 10 & -3.343 & 219.246 & .001 & EA 10 & -0.361 & 252.941 & .718 \\
\hline ES 11 & -3.186 & 259.154 & .002 & SI 11 & -1.725 & 269.062 & .086 & EA 11 & 1.611 & 264.044 & .108 \\
\hline ES 12 & -3.069 & 272.770 & .002 & SI 12 & -1.661 & 256.156 & .098 & EA 12 & -0.567 & 257.250 & .571 \\
\hline ES 13 & -5.153 & 258.382 & .000 & SI 13 & -2.416 & 269.437 & .016 & EA 13 & 3.423 & 286.690 & .001 \\
\hline ES 14 & -2.352 & 256.357 & .019 & SI 14 & -1.167 & 273.236 & .244 & EA 14 & -1.312 & 273.464 & .191 \\
\hline \multirow[t]{3}{*}{ ES 15} & -4.147 & 259.737 & .000 & SI 15 & -5.075 & 239.856 & .000 & EA 15 & 0.223 & 267.758 & .824 \\
\hline & & & & & & & & EA 16 & 1.673 & 273.606 & .096 \\
\hline & & & & & & & & EA 17 & 7.091 & 230.130 & .000 \\
\hline
\end{tabular}

\section{Discusión}

El objetivo principal de esta investigación fue anali- 
zar los niveles de estrés de los alumnos universitarios en un contexto de pandemia derivada por la aparición del COVID-19. Acorde a los resultados hallados en nuestro estudio, los hombres mostraron unos niveles generales de estrés menores a los notificados por las mujeres. De la misma manera, las mujeres también mostraron puntuaciones más elevadas en las dimensiones de estresores y sintomatología asociada al estrés, así como en las subdimensiones de Reacciones físicas, Reacciones psicológicas y Reacciones Comportamentales.

Dichos resultados coinciden con los hallados en trabajos previos como el de Hoyt et al. (2021), los cuales expusieron que el estrés y ansiedad percibidos por el alumnado universitario aumentaron de manera generalizada a raíz de la aparición del COVID-19, añadiendo que las mujeres informaron de un peor bienestar en comparación con los hombres, obteniendo unos resultados similares a los hallados por Wang \& Zhao (2020). Aun así, si atendemos a los resultados extraídos por

En cuanto a la dimensión estrategias de afrontamiento, en el presente trabajo también se observaron diferencias en cuanto a las utilizadas por estudiantes hombres y mujeres. Los hombres recurrieron más frecuentemente a hacer ejercicio físico (EA 13), mientras que las mujeres a escuchar música y ver la televisión (EA 2). En cambio, a la que menos recurrieron ambos sexos fue a la religiosidad (EA 5). De igual manera, otros trabajos de investigación también tuvieron como propósito, analizar cuáles eran las estrategias de afrontamiento de los estudiantes (Baloran 2020; Chandra 2020; Chen, Kaczmarek, y Ohyama 2020). En el caso del trabajo de Chandra (2020), el autor concluyó que se observaron diferencias significativas entre el miedo al fracaso académico y el entorno online y doméstico entre los estudiantes hombres y mujeres. A su vez, se describió que muchos de los estudiantes empezaron a dedicarse a diversas actividades creativas y a seguir cursos que les ayudarán a aprender nuevas habilidades técnicas, utilizando la inteligencia emocional y distanciándose del aburrimiento y de los pensamientos depresivos, con el fin de hacer frente a los efectos negativos derivados de la situación actual de la pandemia. Asimismo, en el contexto francés, otros trabajos refirieron que la ansiedad y síntomas depresivos estaban fuertemente relacionados con las estrategias de afrontamiento, especialmente cuando estas eran de evitación (autodistracción, negación, uso de sustancias, desvinculación conductual, desahogo y autoculpabilización) (Le Vigouroux, Goncalves, y Charbonnier 2021), mostrando resultados en la misma línea de los hallados por Dawson y Golijani-
Moghaddam (2020), en el contexto del Reino Unido.

Por otra parte, los resultados de este trabajo mostraron cierta concordancia en cuanto a los principales estresores identificados tanto en hombres como en mujeres (aunque con unos niveles superiores de estrés en estas últimas) los cuáles son: la sobrecarga de tareas y trabajos académicos (ES 2), la tipología de trabajos requeridos por los profesores (ES 6) y la realización de exámenes, prácticas o trabajos de aplicación (ES 10). En parte, se puede deducir que habitualmente el alumnado en general se ve estresado por este tipo de situaciones, aunque bien es cierto que los altos niveles de estrés reportados por estas tareas pueden verse relacionados con el cambio de las dinámicas para llevarlos a cabo derivadas del contexto pandémico (e.g. dificultades para llevar a cabo trabajos en grupo, nuevas dinámicas de exposición online...). Algunos trabajos previos realizados por diferentes investigadores (Padrón et al. 2021; Pigaiani et al. 2020) expusieron que el malestar psicológico estaba relacionado principalmente con varios estresores, según la percepción de los participantes: el futuro académico, la sobrecarga de tareas, el empeoramiento de los conflictos interpersonales y las restricciones en el contacto social agradable; y mucho menos relacionado con la propagación de la enfermedad y sus consecuencias para la salud física. En cuanto a las estrategias de afrontamiento, en estos casos, tanto las habilidades de reencuadre como las rutinas diarias y las estrategias personales fueron las que se manifestaron como más eficaces.

Por último, en cuanto a los síntomas asociados al estrés, también se mostraron diferencias en cuanto a los indicios más frecuentes y la severidad. En los hombres estudiantes se identificaron la inquietud (SI 7), la dificultad para concentrarse (SI 10) y la desgana por realizar labores académicas (SI 14) como los síntomas más frecuentes. En cambio, en las mujeres estudiantes, se refirieron más frecuentemente a la inquietud (SI 7), la ansiedad, angustia o desesperación (SI 9) y la dificultad para concentrarse (SI 10) como la principal sintomatología asociada al estrés. En este caso, hombres y mujeres compartieron 2 de las 3 principales sintomatologías asociadas al estrés (i.e., SI 7 y SI 10), aunque en las dos coincidencias las mujeres reflejaron un mayor nivel de incidencia que los hombres.

Vistos los resultados expuestos en este trabajo, se puede observar que las medidas adoptadas para la contención del COVID-19 pueden estar afectando a la salud mental de la muestra estudiada, siendo más notorios estos efectos negativos en las mujeres. Estos hallaz- 
gos plantean la necesidad de crear intervenciones eficaces para la reducción de los niveles de estrés en los alumnos universitarios, reduciendo de esta manera el efecto de la pandemia sobre la salud mental de los estudiantes.

Las medidas adoptadas para la contención del virus COVID-19 han repercutido negativamente en muchos aspectos de la vida diaria de las personas, limitando la movilidad y el acceso a centros o actividades deportivas entre otros. Esto se refleja en los resultados de diferentes trabajos de investigación que reflejaron una disminución de los niveles de actividad física en diferentes poblaciones (Caputo y Reichert 2020; Lesser y Nienhuis 2020; Marco-Ahulló et al. 2021). Asimismo, los trabajos realizados en esta línea señalan que aumentar la carga de actividad física puede ser una herramienta interesante para mitigar los efectos de la pandemia sobre la salud mental (Caputo y Reichert 2020). Por tanto, el realizar trabajos de intervención sobre la práctica de ejercicio físico adaptándose a la situación actual, ya sea mediante tele-ejercicio o con planes de entrenamiento que respeten las medidas de seguridad, puede ser de gran interés para intentar suavizar el efecto pandémico sobre variables relacionadas con la salud mental. Como se muestra en los resultados del presente estudio los estudiantes de género masculino ya priorizan la realización de ejercicio físico como principal estrategia de afrontamiento, no siendo así en las estudiantes de género femenino. Por tanto, deberá de tomarse en cuenta este factor, creando además programas de promoción de la práctica de ejercicio físico para este último grupo.

Una vez expuestos y discutidos los resultados, se debe exponer que este estudio no está exento de limitaciones. De hecho, la limitación más importante que se puede encontrar es la imposibilidad de contar con un grupo control que englobase unas características similares al grupo de estudio, pero que no estuviesen viviendo la situación actual generada por el COVID-19, para poder comparar sus resultados. Por ello, mediante este estudio, aunque se describe el estrés académico generado por la pandemia mundial en España, no se puede conocer con certeza cuan distinto hubiese sido dicho estrés si no estuviese dicha pandemia.

En conclusión, el presente estudio ha sido capaz de analizar los niveles de estrés académico y las dimensiones asociadas a este en la población de alumnos universitarios españoles, así como los principales estresores, sintomatología asociada al estrés y estrategias de afrontamiento. Los resultados hallados en este trabajo muestran que las mujeres universitarias muestran unos nive- les de estrés más elevados que sus homólogos de género masculino. Además, se hallaron diferencias significativas entre los grupos de estudiantes en las dimensiones de estresores y sintomatología asociada al estrés, así como en las subdimensiones de reacciones físicas, psicológicas y comportamentales. Sin embargo, no se encontraron diferencias significativas en la dimensión de estrategias de afrontamiento, aunque cada género emplea unas distintas.

\section{Referencias}

Adnan, M., y Anwar, K. 2020. «Online Learning amid the COVID19 Pandemic: Students' Perspectives». Journal of Pedagogical Sociology and Psychology 2(1):45-51.

Agarwal, S., y Kaushik, J. S. 2020. «Student's Perception of Online Learning during COVID Pandemic». Indian Journal of Pediatrics 87(7):554. doi: 10.1007/s12098-020-03327-7.

Alania-Contreras, R. D., Llancari-Morales, R.A., Rafaele de LaCruz, M. R. y Ortega-Révolo, D. I. D. 2020. «Adaptación del cuestionario de estrés académico SISCO SV al contexto de la crisis por COVID-19». Socialium 4(2):111-30. doi: 10.31876/ sl.v4i2.79.

Backoviæ, D.V., Iliæ Zivojinoviæ, J., Maksimoviæ, J. y Maksimoviæ, M. 2012. «Gender Differences in Academic Stress and Burnout among Medical Students in FinalYears of Education». Psychiatria Danubina 24(2):175-81.

Baloran, E.T. 2020. «Knowledge, Attitudes, Anxiety, and Coping Strategies of Students during COVID-19 Pandemic». Journal of Loss and Trauma 25(8):635-42. doi: 10.1080/ 15325024.2020.1769300.

Bao,W. 2020. «COVID-19 and OnlineTeaching in Higher Education: A Case Study of Peking University». Human Behavior and Emerging Technologies 2(2):113-15. doi: https://doi.org/ 10.1002/hbe2.191.

Barraza-Macías, A. 2007. «El Inventario SISCO del Estrés Académico». Investigación Educativa Duranguense (7):90-93.

Barraza-Macías, A. 2018. Inventario SISCO SV-21 Inventario SIStémico COgnoscitivista, para el estudio del Estrés Académico. Segunda versión de 21 Ítems. México, Durango: ECORFAN.

Benham, G. 2020. «Stress and Sleep in College Students Prior to and during the COVID-19 Pandemic». Stress and Health. doi: 10.1002/smi.3016.

Bono, G., Reil, K. y Hescox, J. 2020. «Stress and Wellbeing in Urban College Students in the U.S. during the COVID-19 Pandemic: Can Grit and Gratitude Help?» International Journal ofWellbeing 10(3).

Brougham, R. R., Zail, C. M., Mendoza, C. M. y Miller, J. R. 2009. «Stress, Sex Differences, and Coping Strategies Among College Students». Current Psychology 28(2):85-97. doi: 10.1007/s12144-009-9047-0.

Cabanach, R. G., Fariña Rivera, F., Freire, C., González Berruga, P. y Ferradás, M. M. 2013. «Diferencias en el afrontamiento del estrés en estudiantes universitarios hombres y mujeres». European journal of education and psychology 6(1):19-32.

Caputo, E. L. y Reichert, F. F. 2020. «Studies of Physical Activity 
and COVID-19 During the Pandemic: A Scoping Review». Journal of Physical Activity and Health 17(12):1275-84. doi: 10.1123/jpah.2020-0406.

Chandra,Y. 2020. «Online education during COVID-19: perception of academic stress and emotional intelligence coping strategies among college students». Asian Education and Development Studies 10(2):229-38. doi: 10.1108/AEDS-05-2020-0097.

Chen, E., Kaczmarek, K. y Ohyama, H. 2020. «Student perceptions of distance learning strategies during COVID 19». Journal of Dental Education. doi: 10.1002/jdd.12339.

Cullen, W., Gulati, G. y Kelly, B.D. 2020. «Mental health in the COVID-19 pandemic». QJM: An International Journal of Medicine 113(5):311-12. doi: 10.1093/qjmed/hcaa110.

Dawson, D. L. y Golijani-Moghaddam, N. 2020. «COVID-19: Psychological Flexibility, Coping, Mental Health, andWellbeing in the UK during the Pandemic». Journal of Contextual Behavioral Science 17:126-34. doi: 10.1016/j.jcbs.2020.07.010.

Diez Gutierrez, E. J., y Gajardo Espinoza, K. 2020. «Educating and Evaluating in Times of Coronavirus: The Situation in Spain». Remie-Multidisciplinary Journal of Educational Research 102-34.

Eckles, R. W. 1987. «Stress-Making Friends with the Enemy». Business Horizons 30(2):74-78. doi: 10.1016/00076813(87)90012-7.

García, N. B., y Mazo Zea, R. 2011. «Estrés Académico». Revista de Psicología Universidad de Antioquia 3(2):55-82.

Hoyt, L. T., Cohen, A. K., Dull, B., Castro, E. M. y Yazdani, N. 2021. «»Constant Stress Has Become the New Normal»: Stress and Anxiety Inequalities Among U.S. College Students in the Time of COVID-19». Journal of Adolescent Health 68(2):27076. doi: 10.1016/j.jadohealth.2020.10.030.

Husky, M. M., Kovess-Masfety, V. y Swendsen, J.D. 2020. «Stress and Anxiety among University Students in France during Covid19 Mandatory Confinement». Comprehensive Psychiatry 102:152191. doi: 10.1016/j.comppsych.2020.152191.

Islam, S., Sujan, S. H., Tasnim, R., Sikder, T., Potenza, M.T. y van Os, J. 2020. «Psychological Responses during the COVID-19 Outbreak among University Students in Bangladesh». PLOS ONE 15(12):e0245083. doi: 10.1371/journal.pone.0245083.

Jiménez Hernández, A. S., Cáceres-Muñoz, J. y Martín-Sánchez, M. 2021. «Social Justice, Participation and School during the COVID-19 - The International Project Gira Por La Infancia». Sustainability 13(5):2704. doi: 10.3390/su13052704.

Le Vigouroux, S., Goncalves, A. y Charbonnier, E. 2021. «The Psychological Vulnerability of French University Students to the COVID-19 Confinement». Health Education \& Behavior 48(2):123-31. doi: 10.1177/1090198120987128.

Lesser, I.A., y Nienhuis, C.P. 2020. «The Impact of COVID-19 on Physical Activity Behavior and Well-Being of Canadians». International Journal of Environmental Research and Public Health 17(11):3899. doi: 10.3390/ijerph17113899.

Littlewood, H.. 2014. Mi primer modelamiento de ecuaciones estructurales, 2 a edición.

Marco-Ahulló, A., Montesinos-Magraner, Ll., González, L-M., Morales, J., Bernabéu-García, J. A. y García-Massó, X. 2021. «Impact of COVID-19 on the self-reported physical activity of people with complete thoracic spinal cord injury full-time manual wheelchair users». The Journal of Spinal Cord Medicine 0(0):1-5. doi: 10.1080/10790268.2020.1857490.

Matud, M. P. 2004. «Gender Differences in Stress and Coping
Styles». Personality and Individual Differences 37(7):1401-15. doi: 10.1016/j.paid.2004.01.010.

Miller, S. M. y Kirsch, N. 1987. «Sex differences in cognitive coping with stress». Pp. 278-307 en Gender and stress. New York, NY, US: Free Press.

Misra, R., Crist, M. y Burant, C. J. 2003. «Relationships Among Life Stress, Social Support, Academic Stressors, and Reactions to Stressors of International Students in the United States». International Journal of Stress Management 10(2):137-57. doi: 10.1037/1072-5245.10.2.137.

Moawad, R. A. 2020. «Online Learning during the COVID- 19 Pandemic and Academic Stress in University Students». Revista Româneascã Pentru Educapie Multidimensionalã XII(1 Sup2):100-107.

Padrón, I., Fraga, I., Vieitez, L., Montes, C. y Romero, E. 2021. «A Study on the PsychologicalWound of COVID-19 in University Students». Frontiers in Psychology 12. doi: 10.3389/ fpsyg.2021.589927.

Pfefferbaum, Betty, y Carol S. North. 2020. «Mental Health and the Covid-19 Pandemic». New England Journal of Medicine 383(6):510-12. doi: 10.1056/NEJMp2008017.

Pigaiani,Y., Zoccante, L., Zocca, A., Arzenton, A., Menegolli, M., Fadel, S., Ruggeri, M. y Colizzi, M. 2020. «Adolescent Lifestyle Behaviors, Coping Strategies and SubjectiveWellbeing during the COVID-19 Pandemic: An Online Student Survey». Healthcare 8(4):472. doi: 10.3390/healthcare8040472.

Pomerantz, E. M., Altermatt, E.R. y Saxon, J.L. 2002. «Making the grade but feeling distressed: Gender differences in academic performance and internal distress». Journal of Educational Psychology 94(2):396-404. doi: 10.1037/00220663.94.2.396.

Rogowska, A. M., Kuœnierz, C. y Bokszczanin, A. 2020. «Examining Anxiety, Life Satisfaction, General Health, Stress and Coping Styles During COVID-19 Pandemic in Polish Sample of University Students». Psychology Research and Behavior Management 13:797-811. doi: 10.2147/ PRBM.S266511.

Son, C., Hegde, S., Smith, A., Wang, X. y Sasangohar, F. 2020. «Effects of COVID-19 on College Students' Mental Health in the United States: Interview Survey Study». Journal of Medical Internet Research 22(9):e21279. doi: 10.2196/21279.

Vigo, D., Patten, S., Pajer, K., Krausz, M., Taylor, S., Rush, B., Raviola, G., Saxena, S., Thornicroft, G. yYatham, L.N.. 2020. «Mental Health of Communities during the COVID-19 Pandemic». The Canadian Journal of Psychiatry 65(10):681-87. doi: 10.1177/0706743720926676.

Vindegaard, N. y Benros, M.E. 2020. «COVID-19 Pandemic and Mental Health Consequences: Systematic Review of the Current Evidence». Brain, Behavior, and Immunity 89:531-42. doi: 10.1016/j.bbi.2020.05.048.

Wang, C. y Zhao, H. 2020. «The Impact of COVID-19 on Anxiety in Chinese University Students». Frontiers in Psychology 11. doi: 10.3389/fpsyg. 2020.01168.

Wang, X., Hegde, S., Son, C., Keller, B., Smith, A. y Sasangohar, F. 2020. «Investigating Mental Health of US College Students During the COVID-19 Pandemic: Cross-Sectional Survey Study». Journal of Medical Internet Research 22(9):e22817. doi: $10.2196 / 22817$. 